\title{
New Implementation of Residual Power Series for Solving Fuzzy Fractional Riccati Equation
}

\author{
Moath Ali Alshorman ${ }^{1}$, Nurnadiah Zamri ${ }^{1}$, Mohammed Ali ${ }^{2}$, Asia Khalaf Albzeirat ${ }^{3}$ \\ 1. Faculty of Informatics and Computing, University Sultan Zainal Abidin, Besut, \\ Terengganu, Malaysia \\ 2. Department of Mathematics, Jordan University of Science and Technology, Irbid 22110, \\ Jordan \\ 3. Department of Mathematics, Mutah University, Mutah, Jordan \\ E-mail: alshormanmoath@gmail.com
}

Received: 19 October 2018; Accepted: 14 November 2018; Available online: 25 December 2018

\begin{abstract}
This paper reveals a computational method using a Residual Power Series Method (RPSM) for the solution of fuzzy fractional riccati equation under caputo fractional differentiability. An analytical solution of fuzzy fractional riccati equation is obtained as a convergent fractional power series. The procedure produces solutions of high accuracy, and some illustrative examples are solved with a different value of orders to show the efficiency of the RPSM.
\end{abstract}

Keywords: Fuzzy Fractional Riccati; Residual Power Series Method; Fuzzy Numbers.

\section{Introduction}

Riccati equation was established after the Italian Nobleman Count Jacopo Francesco Riccati (1676-1754) [1]. In the past decades, this type of equation had wide applications in optimal control, random processes, and diffusion problems [2-9]. Currently, in light of the growing fractional concept for the order of differential equation, fractional riccati equation has appeared as a more comprehensive form with a different value of derivative order in many studies [10-15]. Many studies refer to the overlap between the differential equations and the foggy logic, whereby these studies have developed solutions to several fuzzy differential equations by using traditional numerical methods as Laplace transforms [16], transformation method [17], Taylor method [18], Homotopy [19] and Adomian decomposition [20]. Recently, the broader formula of differential equations has included the fractional logic of derivative and the fuzzy logic in its terms. Hence, several studies have developed traditional methods to find solutions to fuzzy fractional differential equations [21-23]. Residual Power Series Method (RPSM) was developed by [24] to solve the fuzzy differential equations and [25] used the same method to solve the Fractional Riccati Equation (FRE) and was extended for the implementation of RPS. Hence, this paper will solve the Fuzzy Fractional Reccati Equation (FFRE). Therefore, we consider the next form of FFRE as follows:

$$
\left\{\begin{array}{c}
D_{t}^{\beta} y(t)+a y(t)+b y(t)=c, 0<\beta \leq 1,0 \leq t \leq R, \\
y(0)=d
\end{array}\right.
$$

Where $a, b$ and $c$ are constants, $d$ is a fuzzy triangular number and $D_{t}^{\beta}$ is the Caputo fractional derivative for order $\beta$. It can be observed that Eq. (1) is a general formulation of FFRE, whereby the initial value $d$ is a fuzzy number.

The rest of the paper is organized as follows: A general introduction about RPS and FFRE is introduced. Section 2 provides the main definitions about fractional calculus, fuzzy numbers. Section 3 presents RPSM for solving FFRE. Section 4 introduces two numerical examples to demonstrate the effectiveness of RPSM. The conclusion of the study is given in section 5 .

\section{Main definitions}

This section contains briefly the main definitions of caputo fractional derivatives and fuzzy numbers. 
Definition 1 [26]. The left Caputo fraction derivative is defined as:

$$
D_{t \in[a, b]}^{c, \beta} y(t)=\frac{1}{\Gamma([\beta]-\beta} \int_{a}^{t}(t-\tau)^{[\beta]-\beta-1} y^{([\beta])} \tau d \tau .
$$

Definition 2 [26]. The right Caputo fraction derivative is defined as:

$$
D_{t \in[a, b]}^{c, \beta} y(t)=\frac{(-1)^{[\beta]}}{\Gamma(\lceil\beta\rceil-\beta} \int_{a}^{t}(t-\tau)^{[\beta]-\beta-1} y^{([\beta])} \tau d \tau .
$$

Definition 3 [27]: Let $u_{F}(t) \in R_{F}^{n}$ and $r \in[0,1]$. The $r-$ cut of $u_{F}(t)$ is the crisp set $\left[u_{F}(t)\right]$ that contains all elements with a membership degree in $u_{F}(t)$ that is greater than or equal to $r$, that is $\left[u_{F}(t)\right]^{r}=\left\{t \in R: u_{F}(t) \geq\right.$ $r$ \}. For a fuzzy interval $u_{F}(t)$, its $r-$ cut is closed and bounded in $R$. These are denoted by:

$$
\left[u_{F}(t)\right]^{r}=\left[u_{1,1 r}(t), u_{1,2 r}(t)\right]
$$

where $u_{1,1 r}=\min \left\{t: t \in\left[u_{F}(t)\right]^{r}\right\}$ and $u_{1,2 r}=\max \left\{t: t \in\left[u_{F}(t)\right]^{r}\right\}$ for each $r \in[0,1]$.

Definition 4 [27]: $u_{F}(t) \in R_{F}, u_{F}$ is triangular if its membership function has the following form:

$$
u_{F}(t)=\left\{\begin{array}{lc}
0, & t<a, \\
\frac{t-a}{b-a}, & a \leq t \leq b, \\
\frac{c-t}{c-b}, & b \leq t \leq c, \\
0, & t>c .
\end{array}\right.
$$

Where it's $r$-cut is simply $\left[u_{F}(t)\right]^{r}=[a+r(b-a), c-r(c-b)]$, for any $r \in[0,1]$.

Definition 5 [28]: A power series expansion of the form

$$
\sum_{m=0}^{\infty} c_{m}\left(t-t_{0}\right)^{m \beta}=c_{0}+c_{1}\left(t-t_{0}\right)^{\beta}+c_{2}\left(t-t_{0}\right)^{2 \beta}+\ldots \ldots,
$$

Where $0 \leq m-1 \prec \beta \leq m, t \leq t_{0}$ is called fractional power series PS about $=t_{0}$.

Theorem 1: Supposes that $f$ has a FPS representation at $t=t_{0}$ of the form

$$
F(t)=\sum_{m=0}^{\infty} c_{m}\left(t-t_{0}\right)^{m \beta}, 0 \leq m-1 \prec \beta \leq m, t_{0} \leq t<t_{0}+R
$$

If $D^{m \beta} f(t)$ is continuous on $\left(t_{0}, t_{0}+R\right)$ for $m \in \mathrm{N}$, then $c_{m}=\frac{D^{m \beta} f\left(t_{0}\right)}{\Gamma(m \beta+1)}$ and $\mathrm{R}$ is the radius of convergence.

Next, the details of the derivation of residual power series solution to the Fractional Riccati Equation are presented.

\section{Residual power series method for solving fuzzy fractional Riccati equations}

For solving FFREs, RPSM is used to solve FREs [25].

Firstly, we consider the new form of general FFREs as follows:

$$
\left\{\begin{array}{c}
D_{t}^{\beta} y(t)+a y(t)+b y(t)=c, 0<\beta \leq 1,0 \leq t \leq R, \\
y(0)=d
\end{array}\right.
$$

Where $0<\delta \leq 1,0 \leq t<\mathrm{R}$, and $d=\left(d_{F_{1}}, d_{F_{2}}, d_{F_{3}}\right)$ is a fuzzy triangular.

By applying the fuzzy set theory, the next form for Eq. (5) is obtained:

$$
\left\{\begin{array}{c}
D_{t}^{\beta} y_{F_{1}}(t)+a y_{F_{1}}(t)+b y_{F_{1}}(t)=c, 0<\beta \leq 1,0 \leq t \leq R \\
D_{t}^{\beta} y_{F_{2}}(t)+a y_{F_{2}}(t)+b y_{F_{2}}(t)=c, 0<\beta \leq 1,0 \leq t \leq R \\
y_{F_{1}}(0)=d_{F_{1}}, y_{F_{2}}(0)=d_{F_{2}}
\end{array}\right.
$$

The RPSM proposes the solution for Eq (6) as Fuzzy Fractional Power Series (FFPS) about the initial point $t=0$ of the form.

$y_{F_{1}}(t)=\sum_{n=0}^{\infty} \frac{a_{F_{1}, n}}{\Gamma(n \beta+1)} t^{n \beta}, y_{F_{2}}(t)=\sum_{n=0}^{\infty} \frac{a_{F_{2}, n}}{\Gamma(n \beta+1)} t^{n \beta}, 0<\delta \leq 1,0 \leq t<R$,

Apparently, according to the fuzzy initial condition using Eq. (4), it yields:

$a_{F_{1}, 0}=\left(d_{F_{2}}-d_{F_{1}}\right) r+d_{F_{1}}$ and $a_{F_{2}, 0}=d_{F_{3}}-\left(d_{F_{3}}-d_{F_{2}}\right) r$ 
Secondly, we let $y_{F_{1}, k}(t) y_{F_{2}, k}(t)$ to denote the $k-t h$ truncated series of $y_{F_{1}}(t)$ and $y_{F_{2}}(t)$ for $k \geq 1$, which can be used to approximate the solution, i.e.

$$
\begin{aligned}
& y_{F_{1}, k}(t)=\left(d_{F_{2}}-d_{F_{1}}\right) r+d_{F_{1}}+\sum_{n=0}^{\infty} \frac{a_{F_{1}, n}}{\Gamma(n \beta+1)} t^{n \beta}, \\
& y_{F_{2}, k}(t)=d_{F_{3}}-\left(d_{F_{3}}-d_{F_{2}}\right) r+\sum_{n=0}^{\infty} \frac{a_{F_{2}, n}}{\Gamma(n \beta+1)} t^{n \beta}, \\
& 0 \prec \beta \leq 1,0 \leq t<R . \beta \leq 1,0 \leq t<R .
\end{aligned}
$$

Thirdly, we define the residual function, $\operatorname{Res}_{y_{F_{1}}}(t)$ and $\operatorname{Res}_{y_{F_{2}}}(t)$ for Eq (6) as:

$$
\begin{aligned}
& \operatorname{Res}_{y_{F_{1}}}(t)=D_{t}^{\beta} y_{F_{1}}(t)+a y_{F_{1}}(t)+b y_{F_{1}}^{2}(t)-c, \\
& \operatorname{Res}_{y_{F_{2}}}(t)=D_{t}^{\beta} y_{F_{2}}(t)+a y_{F_{2}}(t)+b y_{F_{2}}^{2}(t)-c,
\end{aligned}
$$

and accordingly, the $k-t$ residual function, $\operatorname{Res}_{y_{F_{1}}, k}(t)$ and $\operatorname{Res}_{y_{F_{2}}, k}(t)$ is

$$
\begin{aligned}
& \operatorname{Res}_{y_{F_{1}, k}}(t)=D_{t}^{\beta} y_{F_{1}, k}(t)+a y_{F_{1}, k}(t)+b y_{F_{1}, k}^{2}(t)-c, \\
& \operatorname{Res}_{y_{F_{2}, k}}(t)=D_{t}^{\beta} y_{F_{2}, k}(t)+a y_{F_{2}, k}(t)+b y_{F_{2}, k}^{2}(t)-c
\end{aligned}
$$

It's clear that,

$$
\begin{aligned}
& \lim _{k \rightarrow \infty} \operatorname{Res}_{y_{F_{1}}, k}(t)=\operatorname{Res}_{y_{F_{1}}}(t)=0, \\
& \lim _{k \rightarrow \infty} \operatorname{Res}_{y_{F_{2}, k}}(t)=\operatorname{Res}_{y_{F_{2}}}(t)=0
\end{aligned}
$$

for all $t \geq 0$.

By Caputos sense, the fractional derivative of constant function is zero; therefore, $D_{t}^{n \beta} \operatorname{Res}_{y_{F_{1}}}(t)=0$. Also, the fractional derivatives $D_{t}^{n \beta}$ of $\operatorname{Res}_{y_{F_{1}}}(t)$ and $\operatorname{Res}_{y_{F_{1}}, k}(t)$ match at $t=0$ for each $n=0,1,2, \ldots, k$. Also $D_{t}^{n \beta} \operatorname{Res}_{y_{F_{2}}}(t)=0$. Also, the fractional derivatives $D_{t}^{n \beta}$ of $\operatorname{Res}_{y_{F_{2}}}(t)$ and $\operatorname{Res}_{y_{F_{2}}, k}(t)$ match at $t=0$ for each $n=0,1,2, \ldots, k$.

Fourthly, to obtain the value of the coefficient $a_{F_{1}, i}, i=1,2,3, \ldots k$ and $a_{F_{2}, i}, i=1,2,3, \ldots k$ in Eq. (6), we substitute $k$ - th truncated series $y_{F_{1}}(t)$ and $y_{F_{2}}(t)$ into Eq. (7) and using the fact [25].

$$
D_{t}^{(k-1) \beta} \operatorname{Res}_{y_{F_{1}}, k}(0)=0 \text { and } D_{t}^{(k-1) \beta} \operatorname{Res}_{y_{F_{2}}, k}(0)=0,0<\delta \leq 1, k, 1,2,3, \ldots,
$$

we obtain an algebric system in $a_{F_{1}, i}$ and $a_{F_{2}, i}, i=1,2,3, \ldots, k$.

Fifthly, we explicitly apply the previous discussion to find $a_{F_{1}, i}$ and $a_{F_{2}, i}$ under our consideration. first, to determine $a_{F_{1}, 1}$ and $a_{F_{1}, 1}$, we consider $(k=1)$ in (7).

$$
\begin{gathered}
\operatorname{Res}_{y_{F_{1}, 1}}(t)=D_{t}^{\beta} y_{F_{1}, 1}(t)+a y_{F_{1}, 1}(t)+b y_{F_{1}, 1}^{2}(t)-c \\
\operatorname{Res}_{y_{F_{2},}, k}(t)=D_{t}^{\beta} y_{F_{2}, 1}(t)+a y_{F_{2}, 1}(t)+b y_{F_{2}, 1}^{2}(t)-c \\
y_{F_{1}, 1}(t)=\left(d_{F_{2}}-d_{F_{1}}\right) r+d_{F_{1}}+\frac{a_{F_{1}, 1}}{\Gamma(\beta+1)} t^{\beta}, y_{F_{2}, 1}(t)=d_{F_{3}}-\left(d_{F_{3}}-d_{F_{2}}\right) r+\frac{a_{F_{2}, 1}}{\Gamma(\beta+1)} t^{\beta}
\end{gathered}
$$

Therefore,

$$
\begin{aligned}
& \operatorname{Res}_{y_{F_{1}, 1}}(t)=a_{F_{1}, 1}+a\left(a_{F_{1}, 0}+\frac{a_{F_{1}, 1}}{\Gamma(\beta+1)} t^{\beta}\right)+b\left(a_{F_{1}, 0}+\frac{a_{F_{1}, 1}}{\Gamma(\beta+1)} t^{\beta}\right)^{2}-c, \\
& \operatorname{Res}_{y_{F_{2}, 1}}(t)=a_{F_{2}, 1}+a\left(a_{F_{2}, 0}+\frac{a_{F_{2}, 1}}{\Gamma(\beta+1)} t^{\beta}\right)+b\left(a_{F_{2}, 0}+\frac{a_{F_{2}, 1}}{\Gamma(\beta+1)} t^{\beta}\right)^{2}-c .
\end{aligned}
$$

From Eq. (9) we deduce that $\operatorname{Res}_{y_{F_{1}}, 1}(0)=0$ and $\operatorname{Res}_{y_{F_{2}}, 1}(0)=0$. Thus,

$$
a_{F_{1}, 1}=-a_{F_{1}, 0} a-b a_{F_{1}, 0}^{2}+c, a_{F_{2}, 1}=-a_{F_{2}, 0} a-b a_{F_{2}, 0}^{2}+c,
$$

where

$$
a_{F_{1}, 0}=\left(d_{F_{2}}-d_{F_{1}}\right) r+d_{F_{1}}, a_{0}=d \text { and } a_{F_{2}, 0}=d_{F_{3}}-\left(d_{F_{3}}-d_{F_{2}}\right) r .
$$

To obtain $a_{F_{1}, 2}$ and $a_{F_{2}, 2}$, we substitute the 2-nd truncated series 


$$
\begin{gathered}
y_{F_{1}, 2}(t)=a_{F_{1}, 0}+\frac{a_{F_{1}, 1}}{\Gamma(1+\beta)} t^{\beta}+\frac{a_{F_{1}, 2}}{\Gamma(1+2 \beta)} t^{2 \beta}, \\
y_{F_{2}, 2}(t)=a_{F_{2}, 0}+\frac{a_{F_{2}, 1}}{\Gamma(1+\beta)} t^{\beta}+\frac{a_{F_{2}, 2}}{\Gamma(1+2 \beta)} t^{2 \beta}
\end{gathered}
$$

into the second residual function $\operatorname{Res}_{y_{F_{1}}, 2}(t)$ and $\operatorname{Res}_{y_{F_{2}}, 2}(t)$,i.e.,

$$
\begin{aligned}
& \operatorname{Res}_{y_{F_{1}, 2}}(t)=a_{F_{1}, 1}+\frac{a_{F_{1}, 2}}{\Gamma(\beta+1)} t^{\beta}+a\left(a_{F_{1}, 0}+\frac{a_{F_{1}, 1}}{\Gamma(\beta+1)} t^{\beta}+\frac{a_{F_{1}, 2}}{\Gamma(2 \beta+1)} t^{2 \beta}\right)+b\left(a_{F_{1}, 0}+\frac{a_{F_{1}, 1}}{\Gamma(\beta+1)} t^{\beta}+\frac{a_{F_{1}, 2}}{\Gamma(2 \beta+1)} t^{2 \beta}\right)^{2}-c \\
& \operatorname{Res}_{y_{F_{2}, 2}}(t)=a_{F_{2}, 1}+\frac{a_{F_{2}, 2}}{\Gamma(\beta+1)} t^{\beta}+a\left(a_{F_{2}, 0}+\frac{a_{F_{2}, 1}}{\Gamma(\beta+1)} t^{\beta}+\frac{a_{F_{2}, 2}}{\Gamma(2 \beta+1)} t^{2 \beta}\right)+b\left(a_{F_{2}, 0}+\frac{a_{F_{2}, 1}}{\Gamma(\beta+1)} t^{\beta}+\frac{a_{F_{2}, 2}}{\Gamma(2 \beta+1)} t^{2 \beta}\right)^{2}-c
\end{aligned}
$$

By inserting Caputo definition of fractional derivatives $D_{t}^{\beta}$ on both sides of Eq (9) and solving $D_{t}^{\beta} \operatorname{Res}_{y_{F_{1}, 2}}(0)=0$ and $D_{t}^{\beta} \operatorname{Res}_{y_{F_{2}, 2}}(0)=0$ will produce the same result if we just consider the coefficient of the variable $t^{\beta}$ in the expansion of eq (9) and multiply it by $(\beta+1)$. This argument is based on the fact that by Caputo derivative, $D_{t}^{\beta}\left(t^{\beta}\right)=\Gamma(\beta+1)$ and $\left.D_{t}^{\beta}\left(t^{b}\right)\right|_{t=0}=0, b>\beta$. Thus, we get

$$
\begin{aligned}
& a_{F_{1}, 2}=-\left(a+2 b a_{F_{1}, 0}\right) a_{F_{1}, 1} \\
& a_{F_{2}, 2}=-\left(a+2 b a_{F_{2}, 0}\right) a_{F_{2}, 1}
\end{aligned}
$$

Sixthly, finding the other coefficient $a_{F_{1}, k}$ and $a_{F_{2}, k}$ by considering the $k$-th residual function $\operatorname{Res}_{y_{F_{1}, k}}(t)$ and $\operatorname{Res}_{y_{F_{2}, k}}(t)$ and finding the coefficient of the variable $t^{(k-1) \beta}$. The last step is by multiplying the obtained coefficient by the factor leads to the following result for $k \geq 2$

where

$$
\begin{aligned}
& a_{F_{1}, k+1}=\sum_{\substack{i+j=k \\
i, j \in \mathbb{Z}^{+}}} \frac{k \Gamma(k \delta+1)}{\Gamma(i \delta+1) \Gamma(j \delta+1)} a_{F_{1}, i} a_{F_{1}, j}-\left(a+2 b a_{F_{1}, 0}\right) a_{F_{1}, k}, \\
& a_{F_{2}, k+1}=\sum_{\substack{i+j=k \\
i, j \in \mathbb{Z}^{+}}} \frac{k \Gamma(k \delta+1)}{\Gamma(i \delta+1) \Gamma(j \delta+1)} a_{F_{2}, i} a_{F_{2}, j}-\left(a+2 b a_{F_{2}, 0}\right) a_{F_{2}, k},
\end{aligned}
$$

$$
k= \begin{cases}-2 & : i+j \neq k \\ -1 & : i+j=k\end{cases}
$$

\section{Numerical examples}

Example 1: Consider the following FFREs:

$$
\left\{\begin{array}{c}
D_{t}^{\beta} y(t)+a y(t)+b y(t)=c, 0<\beta \leq 1,0 \leq t \leq R, \\
y(0)=d
\end{array}\right.
$$

Where $a=1, b=1, c=1,0<\beta \leq 1,0 \leq t<\mathrm{R}, d=(0,0.25,0.5)$ is a fuzzy triangular number and $\beta=$ $1.0,0.9,0.8,0.7$ and 0.6 for $r=0$.

By applying the fuzzy theory on Eq. (14), we obtain the following system:

$$
\left\{\begin{array}{c}
D_{t}^{\beta} y_{F_{1}}(t)+a y_{F_{1}}(t)+b y_{F_{1}}(t)=c, 0<\beta \leq 1,0 \leq t \leq R, \\
D_{t}^{\beta} y_{F_{2}}(t)+a y_{F_{2}}(t)+b y_{F_{2}}(t)=c, 0<\beta \leq 1,0 \leq t \leq R, \\
y_{F_{1}}(0)=d_{F_{1}}, y_{F_{2}}(0)=d_{F_{2}},
\end{array}\right.
$$

where $a=1, b=1, c=1,0<\beta \leq 1,0 \leq t<\mathrm{R}, d_{F_{1}}=0, d_{F_{2}}=0.5 \quad \beta=1.0,0.9,0.8,0.7$ and 0.6. at $r=0$.

To solve the Eq. (14), we used the proposed steps in previous section with 10 terms. Then the approximate results obtained are compared with the exact solutions at $\beta=1$ that are presented in Tables 1-2, where the exact solutions for $y_{F_{1}}(t)$ and $\mathrm{r} y_{F_{2}}(t)$ where $\beta=1$ is given by:

$y_{F_{1}}(t)=-\frac{(-1+\sqrt{5})\left(-1+e^{\sqrt{5} x}\right)}{-3+\sqrt{5}-2 e^{\sqrt{5} x}}, y_{F_{2}}(t)=\frac{0.5\left(-0.180339+1.236068 e^{\sqrt{5} x}\right)}{0.055728+e^{\sqrt{5} x}}$. 
Table 1: Numerical solution for $y_{F_{1}}(t), \beta=1.0,0.9,0.8,0.7$ and $0.6, r=0$

\begin{tabular}{cccccccc}
\hline$x$ & Exact & \multicolumn{5}{c}{ Approximate } & Errors \\
\cline { 3 - 7 } & & $\beta=1.0$ & $\beta=0.9$ & $\beta=0.8$ & $\beta=0.7$ & $\beta=0.6$ & \\
\hline 0.0 & 0.0 & 0.0 & 0.0 & 0.0 & 0.0 & 0.0 & 0.0 \\
0.1 & 0.094862 & 0.094862 & 0.121163 & 0.152119 & 0.187030 & 0.224376 & $1.705302566 \times 10^{-13}$ \\
0.2 & 0.179113 & 0.179113 & 0.210076 & 0.241790 & 0.272633 & 0.301079 & $3.28810118 \times 10^{-10}$ \\
0.3 & 0.252691 & 0.252691 & 0.281149 & 0.307244 & 0.329889 & 0.348751 & $2.663535387 \times 10^{-8}$ \\
0.4 & 0.316007 & 0.316007 & 0.338561 & 0.357050 & 0.371259 & 0.382722 & $5.878406331 \times 10^{-7}$ \\
0.5 & 0.369806 & 0.369800 & 0.385052 & 0.395671 & 0.402207 & 0.411177 & $6.355153427 \times 10^{-6}$ \\
\hline
\end{tabular}

Table 2: Numerical solution for $y_{F_{2}}(t), \beta=1.0,0.9,0.8,0.7$ and $0.6, r=0$

\begin{tabular}{cccccccc}
\hline$x$ & Exact & \multicolumn{5}{c}{ Approximate } & \multirow{2}{*}{ Errors } \\
\cline { 3 - 7 } & & $\beta=1.0$ & $\beta=0.9$ & $\beta=0.8$ & $\beta=0.7$ & $\beta=0.6$ & \\
\hline 0.0 & 0.25 & 0.25 & 0.25 & 0.25 & 0.25 & 0.25 & 0.0 \\
0.1 & 0.522642 & 0.522642 & 0.528400 & 0.534856 & 0.541727 & 0.548643 & $7.327471963 \times 10^{-15}$ \\
0.2 & 0.541098 & 0.541098 & 0.547106 & 0.552879 & 0.558148 & 0.562752 & $1.485223056 \times 10^{-11}$ \\
0.3 & 0.556086 & 0.556086 & 0.560954 & 0.565095 & 0.568452 & 0.571061 & $1.257874249 \times 10^{-9}$ \\
0.4 & 0.568222 & 0.568222 & 0.571513 & 0.573946 & 0.575604 & 0.5764820 & $2.91083696 \times 10^{-8}$ \\
0.5 & 0.578025 & 0.578025 & 0.579699 & 0.580583 & 0.5806833 & 0.5796343 & $3.307333044 \times 10^{-7}$ \\
\hline
\end{tabular}

Tables 1 and 2 show a comparison of the approximate solution of $y_{F_{1}, 10}(t)$ and $y_{F_{2}, 10}(t)$ of different values of the fractional Caputo derivative order $0<\beta \leq 1.0$ with exact solutions at $\beta=1.0$. It is clear in Tables 1 and 2 that the approximate solutions is in high agreement with the exact solution at $\beta=1$

Example 2: Consider the following FREs:

$$
\left\{\begin{array}{c}
D_{t}^{\beta} y(t)+a y(t)+b y(t)=c, 0<\beta \leq 1,0 \leq t \leq R, \\
y(0)=d
\end{array}\right.
$$

Where $a=0.75, b=0.5, c=0.75,0<\beta \leq 1,0 \leq t<\mathrm{R}, d=(0,0.1,0.2)$ is a fuzzy triangular number and $\beta=1.0,0.9$, $0.8,0.7$ and 0.6 for $r=0$.

By applying the fuzzy theory on Eq. (15), we obtain the following system:

$$
\left\{\begin{array}{c}
D_{t}^{\beta} y_{F_{1}}(t)+a y_{F_{1}}(t)+b y_{F_{1}}(t)=c, 0<\beta \leq 1,0 \leq t \leq R \\
D_{t}^{\beta} y_{F_{2}}(t)+a y_{F_{2}}(t)+b y_{F_{2}}(t)=c, 0<\beta \leq 1,0 \leq t \leq R \\
y_{F_{1}}(0)=d_{F_{1}}, y_{F_{2}}(0)=d_{F_{2}}
\end{array}\right.
$$

where $a=0.75, b=0.5, c=0.5,0<\beta \leq 1,0 \leq t<\mathrm{R}, d_{F_{1}}=0, d_{F_{2}}=0.2 \quad \beta=1.0,0.9,0.8,0.7$ and 0.6 at $r=0$.

To solve the Eq. (16), we used the proposed steps in previous section with 10 terms. Then the approximate results obtained are compared with the exact solutions at $\beta=1$ that are presented in Table 3 and 4 , where the exact solutions for $y_{F_{1}}(t)$ and $y_{F_{2}}(t)$ whereby $\beta=1$ given by:

$$
y_{F_{1}}(t)=\frac{0.686141\left(-1 .+1 \times 2.718281^{1.436141 x}\right)}{0.313859+2.718282^{1.436141 x}}, \quad y_{F_{2}}(t)=\frac{0.686141\left(-0.649129+2.71828182^{1.436141 x}\right)}{0.2037351+2.718282^{1.436141 x}} \text {. }
$$

Table 3: Numerical solution for $y_{F_{1}}(t), \beta=1.0,0.9,0.8,0.7$ and $0.6, r=0$

\begin{tabular}{cccccccc}
\hline$x$ & Exact & \multicolumn{5}{c}{ Approximate } & \multirow{2}{*}{ Errors } \\
\cline { 3 - 7 } & & $\beta=1.0$ & $\beta=0.9$ & $\beta=0.8$ & $\beta=0.7$ & $\beta=0.6$ & \\
\hline 0.0 & 0.0 & 0.0 & 0.0 & 0.0 & 0.0 & 0.0 & 0.0 \\
0.1 & 0.072169 & 0.072169 & 0.092834 & 0.117759 & 0.146905 & 0.179714 & $5.828670879 \times 10^{-16}$ \\
0.2 & 0.138649 & 0.138649 & 0.164649 & 0.192593 & 0.221530 & 0.250307 & $1.17919563 \times 10^{-12}$ \\
0.3 & 0.199482 & 0.199482 & 0.225647 & 0.251470 & 0.275959 & 0.298287 & $9.118489297 \times 10^{-11}$ \\
0.4 & 0.254809 & 0.254809 & 0.278310 & 0.299786 & 0.31858 & 0.334436 & $1.914250203 \times 10^{-9}$ \\
0.5 & 0.304852 & 0.304852 & 0.324092 & 0.340273 & 0.353210 & 0.363249 & $1.957893259 \times 10^{-8}$ \\
\hline
\end{tabular}


Table 4: Numerical solution for $y_{F_{2}}(t), \beta=1.0,0.9,0.8,0.7$ and $0.6, r=0$

\begin{tabular}{cccccccc}
\hline$x$ & Exact & \multicolumn{5}{c}{ Approximate } & \multirow{2}{*}{ Errors } \\
\cline { 3 - 6 } & & $\beta=1.0$ & $\beta=0.9$ & $\beta=0.8$ & $\beta=0.7$ & $\beta=0.6$ & \\
\hline 0.0 & 0.2 & 0.2 & 0.2 & 0.2 & 0.2 & 0.2 & 0.0 \\
0.1 & 0.255279 & 0.255279 & 0.270827 & 0.289377 & 0.310773 & 0.334470 & $1.665334537 \times 10^{-16}$ \\
0.2 & 0.305276 & 0.305276 & 0.324332 & 0.344498 & 0.365028 & 0.385093 & $1.38811184810^{-12}$ \\
0.3 & 0.350269 & 0.350269 & 0.368931 & 0.387014 & 0.403837 & 0.418914 & $1.201665434 \times 10^{-11}$ \\
0.4 & 0.390577 & 0.390577 & 0.406840 & 0.421376 & 0.433823 & 0.444127 & $2.839071367 \times 10^{-9}$ \\
0.5 & 0.426544 & 0.426544 & 0.439359 & 0.449827 & 0.457954 & 0.464077 & $3.289613287 \times 10^{-8}$ \\
\hline
\end{tabular}

Tables 3 and 4 show a comparison of the approximate solution of $y_{F_{1}, 10}(t)$ and $y_{F_{2}, 10}(t)$ of different values of the fractional Caputo derivative order $0<\beta \leq 1.0$ with exact solutions at $\beta=1.0$. It is clear through Tables 3 and 4 that the approximate solution is in high agreement with the exact solution at $\beta=1$

\section{Conclusions}

In this paper, we have studied the solutions of FFREs with Caputo derivatives by RPSM. The proposed steps are considerably convenient since it requires less effort and does not need a complex software for the application proposed procedure of solution. The accuracy of results obtained in this paper from the illustrated two examples indicates the effectiveness of the method. It also refers to the possibility of future research to find solutions to various forms of fuzzy fractional equations by using RPSM.

\section{References}

[1] Reid, William Thomas. Riccati differential equations. Elsevier, 1972.

[2] Lasiecka, Irena. Differential and algebraic Riccati equations with application to boundary/point control problems: continuous theory and approximation theory. LN in Control and Information Sciences 164 (1991).

[3] Zhou, Kemin, John Comstock Doyle, and Keith Glover. Robust and optimal control. Vol. 40. New Jersey: Prentice hall, 1996.

[4] Benner, Peter, Jing-Rebecca Li, and Thilo Penzl. Numerical solution of large-scale Lyapunov equations, Riccati equations, and linear-quadratic optimal control problems. Numerical Linear Algebra with Applications 15, no. 9 (2008): 755-777.

[5] Yong, Jiongmin. Linear-quadratic optimal control problems for mean-field stochastic differential equations. SIAM journal on Control and Optimization 51, no. 4 (2013): 2809-2838.

[6] El Karoui, Nicole, Shige Peng, and Marie Claire Quenez. Backward stochastic differential equations in finance. Mathematical finance 7, no. 1 (1997): 1-71.

[7] Raja, Muhammad Asif Zahoor, Junaid Ali Khan, and Ijaz Mansoor Qureshi. A new stochastic approach for solution of Riccati differential equation of fractional order. Annals of Mathematics and Artificial Intelligence 60, no. 3-4 (2010): 229-250.

[8] Bittanti, Sergio, Alan J. Laub, and Jan C. Willems, eds. The Riccati Equation. Springer Science \& Business Media, 2012.

[9] Suazo, Erwin, Sergei K. Suslov, and José M. Vega-Guzmán. The Riccati differential equation and a diffusion-type equation. New York J. Math 17 (2011): 225-244.

[10] Momani, Shaher, and Nabil Shawagfeh. Decomposition method for solving fractional Riccati differential equations. Applied Mathematics and Computation 182, no. 2 (2006): 1083-1092.

[11] Odibat, Zaid, and Shaher Momani. Modified homotopy perturbation method: application to quadratic Riccati differential equation of fractional order. Chaos, Solitons \& Fractals 36, no. 1 (2008): 167-174.

[12] Cang, Jie, Yue Tan, Hang Xu, and Shi-Jun Liao. Series solutions of non-linear Riccati differential equations with fractional order. Chaos, Solitons \& Fractals 40, no. 1 (2009): 1-9.

[13] Zhang, S. A generalized Exp-function method for fractional Riccati differential equations. (2010): 48-51.

[14] Merdan, Mehmet. On the solutions fractional Riccati differential equation with modified RiemannLiouville derivative. International Journal of differential equations 2012 (2012).

[15] Sakar, Mehmet Giyas, Ali Akgül, and Dumitru Baleanu. On solutions of fractional Riccati differential equations. Advances in Difference Equations 2017, no. 1 (2017): 39. 
[16] Salahshour, S., T. Allahviranloo, and S. Abbasbandy. Solving fuzzy fractional differential equations by fuzzy Laplace transforms. Communications in Nonlinear Science and Numerical Simulation 17, no. 3 (2012): 1372-1381.

[17] Allahviranloo, Tofigh, Narsis Aftab Kiani, and N. Motamedi. Solving fuzzy differential equations by differential transformation method. Information Sciences 179, no. 7 (2009): 956-966.

[18] Abbasbandy, Saeid, and Tofigh Allah Viranloo. Numerical solutions of fuzzy differential equations by Taylor method. Computational Methods in Applied Mathematics Comput. Methods Appl. Math. 2, no. 2 (2002): 113-124.

[19] Salah, Ahmed, Majid Khan, and Muhammad Asif Gondal. A novel solution procedure for fuzzy fractional heat equations by homotopy analysis transform method. Neural Computing and Applications 23, no. 2 (2013): 269-271.

[20] Paripour, Mahmoud, Elahe Hajilou, Afshin Hajilou, and Homa Heidari. Application of Adomian decomposition method to solve hybrid fuzzy differential equations. Journal of Taibah University for Science 9, no. 1 (2015): 95-103.

[21] Salahshour, Soheil, Ali Ahmadian, Norazak Senu, Dumitru Baleanu, and Praveen Agarwal. On analytical solutions of the fractional differential equation with uncertainty: application to the Basset problem. Entropy 17, no. 2 (2015): 885-902.

[22] Albzeirat, Asia Khalaf, Muhammad Zaini Ahmad, Shaher Momani, and Banan Maayah. Numerical solution of second-order fuzzy differential equation of integer and fractional order using reproducing kernel hilbert space method tools. Far East Journal of Mathematical Sciences 101, no. 6 (2017): 1327.

[23] Albzeirat, Asia Khalaf, Muhammad Zaini Ahmad, Shaher Momani, and Israr Ahmad. New implementation of reproducing kernel Hilbert space method for solving a fuzzy integro-differential equation of integer and fractional orders. Journal of King Saud University-Science 30, no. 3 (2018): 352-358.

[24] Arqub, O. Abu. Series solution of fuzzy differential equations under strongly generalized differentiability. Journal of Advanced Research in Applied Mathematics 5, no. 1 (2013): 31-52.

[25] Ali, Mohammed, Imad Jaradat, and Marwan Alquran. New computational method for solving fractional Riccati equation. J Math Comput Sci 17, no. 1 (2017): 106-114.

[26] Caputo, Michele. Linear models of dissipation whose Q is almost frequency independent - II. Geophysical Journal International 13, no. 5 (1967): 529-539.

[27] Albzeirat, Asia Khalaf, Israr Ahmad, Muhammad Zaini Ahmad, Shaher Momani, and Fathilah Mohammad Alipiah. Generalizing the meaning of derivatives and integrals of any order differential equations by fuzzy-order derivatives and fuzzy-order integrals. Journal of King Saud University-Science (2017). https://doi.org/10.1016/j.jksus.2017.12.014

[28] Moaddy, Khaled, Mohammed Al-Smadi, and Ishak Hashim. A novel representation of the exact solution for differential algebraic equations system using residual power-series method. Discrete Dynamics in Nature and Society 2015 (2015). http://dx.doi.org/10.1155/2015/205207

(C) 2018 by the author(s). This work is licensed under a Creative Commons Attribution 4.0 International License (http://creativecommons.org/licenses/by/4.0/). Authors retain copyright of their work, with first publication rights granted to Tech Reviews Ltd. 